This article has been published in:

Journal of Pragmatics 58 (2013) 87-107

Elsevier

http://dx.doi.org/10.1016/j.pragma.2013.05.014

If you want to quote from this document, please consult the page numbers in the right hand margins.

\title{
The role of emotions in relational work
}

\author{
Andreas Langlotz, Miriam A. Locher
}

\begin{abstract}
While the role of emotions for communication has been recognized as important in numerous research disciplines, insights have rarely been exploited for linguistic research, nor have they been incorporated systematically in the traditional theories on relational work. This paper offers a literature review on emotion research for linguists and then focuses in particular on the creation of relational meaning within interpersonal pragmatics. Since emotional display is often signalled in gestures or facial expressions in addition to or in complement to linguistic evidence, we propose taking a multi-modal approach to the study of relationship construction. For this purpose we combine Clark's (1996) work on the creation of meaning with a multi-modal tool-kit for analysis. The paper ends with an assessment on how this inclusion of emotional cues in our analysis of relational work improves our understanding of interpersonal pragmatics.
\end{abstract}

\section{Introduction}

This paper explores the emotional display of a linguistic and multi-modal nature within the framework of interpersonal pragmatics. In recent years, traditional politeness research has opened up its focus to concentrate more generally on the relational and interpersonal aspect of communication, including face-aggravating, face-maintaining and face-enhancing behaviour (see Locher, 2013 for an overview). The discursive approach to the study of politeness, by no means a unified approach (see Locher, 2012; LPRG Group, 2011), highlights that there is a difference between emic and etic judgments on what is deemed polite, impolite, uncouth, etc. One of the research gaps within this research tradition was identified as the question of how judgements by interactants come about. Next to stating 
that judgments on whether a particular utterance was deemed polite or impolite, etc. depend on situated assessments of the exploitation of norms in situ, it was pointed out that these assessments crucially also have an emotional component (Locher and Langlotz, 2008; Culpeper, 2011; Spencer-Oatey, 2011) in that interactors react with emotions to the violations or the adherence of personal expectations and social norms. This link is also addressed by Spencer-Oatey (2007:644) who claims that "[F]ace is associated with affective sensitivity" and that "face is a vulnerable phenomenon, and hence associated with emotional reactions." However, she also concedes that emotions have rather been "[a]n implicit thread running through nearly all this 'relational' research" and, following Culpeper (2011) and Ruhi (2009), she therefore states that "there has been surprisingly little research on this aspect, at least until recently" (Spencer-Oatey, 2011:3568). This raises the question of how emotions can be included into the theoretical and methodological picture of interpersonal pragmatics and of how their role in relational work can be theorized and explored. Since this aspect has not been systematically pursued yet within (im)politeness research, this paper takes this research gap as a starting point to review the existing literature on emotions in an endeavour to make the research insights usable for interpersonal pragmatics.

The paper is structured as follows. In section 2 we will first position our approach to 88 relational work within the context of interpersonal pragmatics to set the stage for the subsequent theoretical considerations on the emotional impact on relational work. Having identified the emotional gap in this linguistic research strand, we will then provide an overview of previous research into language and emotion in linguistics as well as communication studies (sections 3-5). While emotions have not played a crucial role in the former, research on emotional communication is far more advanced in the latter. Although we cannot present an extensive literature review of either area, our aim is to discuss to what extent previous insights can be integrated into the framework of relational work and in how far our approach can be seen as making an independent contribution to the complex connections between language and emotion from a decidedly interpersonal pragmatic perspective. For this purpose the literature review will be organized around three central questions:

- What are emotions? (section 3)

- What are the links between emotions and interpersonal relationships? (section 4)

- How are emotions signalled in interaction and how is the communication of emotions influenced by social and cultural norms? (section 5)

In line with these questions, we aim to distill the relevant parameters for an analysis of emotions within the context of relational work from previous work. To exemplify our own approach to analysing emotion and relational work, we will employ one example of a brief interaction between two characters from the cartoon 'Calvin and Hobbes'. This data is introduced in section 6 with a focus on the multi-modal emotional signals and their potential evaluative meanings that influence the dynamic construal of the relationship between the protagonists. In section 7 we will then focus on the dynamic practice of 
signalling emotions and of how these multi-modal signals may lead to the construal of evaluative meanings that affect the given relationship. With reference to Clark's (1996:Ch. 6) model of communicative signalling, we will first focus on the methodological challenges of handling composite emotional signals and then discuss how the interactors employ these signals to coordinate integrated evaluations of social relationships. The 'Calvin and Hobbes' cartoon will thus serve to establish the link between our theoretical considerations and their implementation in data analysis. The paper concludes with providing some further suggestions for modelling emotional contributions in a discursive approach to relational meaning.

\section{Interpersonal pragmatics, relational work and the emotional lacuna in linguistic analysis}

To position our approach, we first need to define Interpersonal Pragmatics and Relational Work and point to key issues within this approach. The relational function of language use is of central interest to the domain of Interpersonal Pragmatics:

The term 'interpersonal pragmatics' is used to designate examinations of interactions between people that both affect and are affected by their understandings of culture, society, and their own and others' interpretations. (Locher and Graham, 2010:2)

Interpersonal Pragmatics is not a new field per se nor is it a new theory, but it highlights the interpersonal/relational perspective on language in use. In line with this perspective, social meaning is created as interpersonal relationships are discursively constructed. In this process, the interactors perform and negotiate situated social images and roles. In doing so, relationships are created, maintained, and challenged through interaction. Interpersonal pragmatics thus casts particular light on relational work (Locher, 2004; Locher and Watts, 2005, 2008).

Being embedded in the traditions of interpersonal pragmatics and (im)politeness ${ }^{1}$ research, relational work positions itself as a discursive approach to the management of relational meaning, i.e. the meaning that we attribute to social relationships.

Relational work refers to all aspects of the work invested by individuals in the construction, maintenance, reproduction and transformation of interpersonal relationships among those engaged in social practice. (Locher and Watts, 2008:96)

Thus, the notion of relational work considers relationships as dynamic constructs that emerge through interaction in situated contexts and relative to norms. In order to mediate the discursive construction of relational meaning, interlocutors depend on processes of multi-modal signalling that are distributed over dynamic action-sequences (see Clark, ^ 1996). It is

\footnotetext{
1 Please consult Locher $(2008,2012)$ for a discussion of how judgments of politeness, impoliteness, rudeness or any other first order judgement by interactants is linked to relational work and identity construction.
} 
these complex and interdependent processes of signalling and signal interpretation that characterize the 'work' invested by the interactional partners. Crucially, however, relational work is not only guided by the interactors' personal 'work' decisions. Interactions are embedded in socio-normative contexts that influence the ways in which the communicators choose their ways of signalling and how they interpret them.

It is further necessary to highlight the socio-cognitive dimensions of relational work. Both interactants must draw on their knowledge and sense-making skills to interpret their communicative actions. They do this by activating frames for interactional conventions, roles, and procedures (Tannen, 1993:53). They signal, construct, interpret, and assign activity-specific goals and intentions (see, e.g. Tomasello et al., 2005); and they engage in situated processes of conceptualization, i.e. cognitive processes of inferring meaning in situ (Langlotz, 2010). It is important to emphasize that these sense-making processes are not cognitively isolated nor socially encapsulated in the individual. Rather, through the interaction, the interlocutors' cognitive work becomes interdependent. Note that this does not imply that the individual mind does not constitute the medium for cognitive processes (see Spencer-Oatey's criticism in this volume). However, the discursive acts of one partner are adapted and designed to influence and 'manipulate' the mental states of the other. As a consequence, the scope of the partners' cognitive processing in terms of setting goals, planning discourse moves, and taking communicative decisions is social, rather than personal (see Arundale, 2010).

As mentioned above, the study of relational work and linguistics in general has hardly integrated the systematic analysis of its emotional component into its research scope. While the existence of emotive language or affective meaning had already been recognized by Aristotle (McKeon, 1941) and emotions play a central role in human psychology and sociality, the contribution of emotional framing for sense-making processes has not been extensively theorized nor thoroughly researched in linguistics apart from a number of explorations into this field. In structuralism and generativism emotions played a highly marginal if not inexistent role (Jay and Janschewitz, 2007). For example, while the idea of word connotation in structural semantics points to the existence of the emotional associations that people have for lexemes, the theory of langue clearly put its emphasis on the denotation of words and thus imposed a theoretical and terminological boundary between linguistic and emotional analysis (Schwarz-Friesel, 2007:162-173). In generativism such epistemological boundaries were drawn on a more cognitive level: Defending a highly modular view of cognitive and linguistic competence, generativism clearly separated linguistic knowledge from all other psychological phenomena. As a consequence, emotional aspects do not have a place in the generative view of language.

Emotional dimensions of language have been more openly addressed in the functional traditions of linguistic analysis. Back in 1934, Bühler included an expressive function into his organon model of language-based communication. The same is true for Jakobson's (1960) model of the six language functions, which attributes an emotive function of expression to the speaker. While being fairly restricted to the analysis of individual 
utterances, these early functionalist frameworks have certainly paved the way for a more integrative and comprehensive view of language and linguistic meaning. This, for instance, is evidenced by the fact that Halliday (1975) makes a tripartite distinction between ideational, interpersonal, and textual functions of linguistic units in the context of his systemic-functional grammar. Interestingly, however, emotional components still seem to play a minor role in this functional classification as part of the interpersonal function.

More integrative approaches to the relationship between language and emotion have been offered in anthropological linguistics and linguistic ethnography (see Wilce, 2009 for an overview), conversation and discourse analysis (see Pepin, 2008 for an overview), and cognitive linguistics (for overviews see Niemeier and Dirven, 1997; Foolen et al., 2012). ${ }^{2}$ Extensive research on emotional communication has been offered in communication studies. In what follows, we will engage with these insights from previous research to discuss to what extent they are conducive to the analysis of relational work. The aim of the literature review is to distill essential parameters for a theoretical and methodological framework that allows us to incorporate the analysis of emotions in relational work. We will start by discussing the fundamental role of emotions in general and ask how emotions can become an object of analysis for linguistics in general and interpersonal pragmatics in particular.

\section{What are emotions?}

Although emotions are empirically real phenomena that we experience on an everyday basis, they have so far escaped the grasp of science. Adopting a highly sceptical philosophical perspective, Griffiths (1997:247) goes as far as to deny emotions the status of natural categories altogether: "The research surveyed in this book suggests that the general concept of emotion has no role in any future psychology." Instead he claims that what we perceive as emotional states and what we denote by the word emotion may involve a heterogeneous cluster of inner psychic states.

Taking the intricate nature and the diverse functions of emotions into account, 90 Schwarz-Friesel (2007) defines them as follows:

Emotions are multi-dimensional, internally represented and subjectively experienced syndrome categories; they can be self-perceived by an individual on the introspective planes of the mind as well as the body; their experiential values are associated with a positive or negative judgement; and they can be expressed to others in the form of perceptible display variants. The judgmental processes concern evaluations through which an individual appraises his/her own bodily sensations, psychological state, behavioural impulses, cognitive representations or general environmental states (in the broadest sense). (Schwarz-Friesel, 2007:55, our translation)

\footnotetext{
2 Recent international research incentives have also been interested in the link between language and emotion such as the Languages of Emotion Cluster of Excellence at the Freie Universität Berlin (http://www.languages-of-emotion.de/).
} 
Thus, Schwarz-Friesel describes emotions as syndrome categories that comprise different facets: internal mental and body states, perceptible display variants, and judgmental processes that lead to subjective evaluations of the inner and outer world of experience.

In psychology, the complex nature of emotions and the difficulty of delimiting them as psychological entities has led to process models that regard them as a dynamic assembly of multiple components. According to Planalp (1999:11) a process theory of emotion involves the following dimensions: "(1) objects, causes, precipitating events, (2) appraisal, (3) physiological changes, (4) action tendencies/action/expression, (5) regulation" (emphasis in original). For instance, a basic emotion such as fear may be caused by an exam situation (1), which is appraised as being too difficult (2), and thus leads to physiological changes such as an increased heart rate, nervousness, sweating, etc. (3). This might lead to certain action tendencies such as stuttering, bodily agitation, or a shaky handwriting, and may be expressed to the examiner by showing a concerned face, by lowering the voice, or by directly addressing one's state verbally: "I'm feeling so nervous" (4). The state of fear can also be regulated by the examinee's trying to be brave or by the examiner's attempts at relaxing the student (5). While different approaches to emotion may highlight one of the components from this emotional process more than others, we can assume that emotions function as internally represented value-categories that can be perceived subjectively and expressed to interactors. Hence, emotions must not be seen as primitive and irrational psychic states, but as a complex, embodied value system (Damasio, 1994, 1999, 2003).

Along these general lines emotions fulfil evaluative functions that help us define our relationship to and our understanding of the world of experience (cf. Schwarz-Friesel, 2007:67). Emotions influence our reactions to and our actions upon our physical and social environments. They may be directed towards other people, objects, states, events, etc. and thus help us define our relationship to them. And self-referential emotions such as shame, regret or pride define the relationships to our selves. Very strong emotional states like panic, fear, lust may cause overwhelming bodily symptoms that determine our orientation towards and further engagement with our world of experience. On the basis of these dimensions, Table 1 proposes a grid for the description and categorization of emotions (see Schwarz-Friesel, 2007). For instance, panic can be described as a form of the basic emotion type fear that is caused by situational factors and causes very strong bodily symptoms. It is associated with a negative evaluation and is very intensive but momentary and occasional rather than permanent. By contrast, sorrow is an intensive and permanent negative emotion that is a form of sadness and is self-referential although it is triggered by the loss of a close friend or relative.

Different instantiations of the central components of the emotional process lead to different emotion types (Damasio, 1994:Ch. 7). Primary emotions (anger, fear, happiness, etc.) are seen as continuing the biological legacy of life preservation that we share with other animals (Darwin, 1872). They are triggered by external stimuli and, through innate dispositional representations, evoke a body state (increased heart rate, blood pressure, etc.) that corresponds to the primary emotion. The search for basic, primary emotions is 
reflected in various psychological studies (Ekman, 2003; Plutchik, 2003). Most prominently, the psychologist Ekman $(1973,2003)$ distinguishes the following basic and universal types of emotions (and their corresponding facial expressions, see section 5): Anger, fear, disgust, surprise, happiness, sadness. However, the search for a universal set of basic and innate primary human emotions has not led to conclusive results and has remained fairly controversial as emotions and their expression seem to be subject to strong cultural differences (for an overview see Planalp, 1999:Ch. 7). Most importantly, beyond the potential set of basic emotion types,

Table 1. Classificatory grid for emotion categories, based on Schwarz-Friesel (2007:Ch. 3.3).

\begin{tabular}{ll}
\hline Emotion & \\
\hline A. Type and function: & Emotion categories \\
B. Quality: & Positive vs. negative evaluation \\
C. Intensity: & Strong - weak continuum \\
D. Duration: & Permanent vs. temporary \\
\hline
\end{tabular}

there are a great many emotional subtypes, some of which are highly culture-specific. The strong influence of social and cultural learning on emotion management is captured by the term secondary emotion (Damasio, 1994:134-139). Unlike primary emotions, secondary emotions (embarrassment, guilt, pride, etc.) are learned through experience and socialization (Turner and Stets, 2005:15-19; see also Culpeper, 2011:59).

The socially constructed and culture-specific nature of emotions has been most prominently discussed by researchers interested in the cultural relativity of human concepts, behaviours, practices, and languages. As sketched above, Western psychologists and neuropsychologist have assumed emotions to have strong biological foundations. They have therefore promoted a highly universalist view of human emotionality. By contrast, anthropological linguists have highlighted the strong cultural relativity of emotion concepts, emotion words, and emotional practices of expression and communication (see, e.g. Goddard, 2002; Wierzbicka, 1994). Along similar lines and promoting a social constructionist approach to emotion, Harré claims that:

[...], the overlay of cultural and linguistic factors on biology is so great that the physiological aspect of some emotional states has had to be relegated to secondary status, as one among the effects of the more basic sociocultural phenomena. (Harré, 1986a,b:4)

Highlighting the socially constructed nature of emotions over their presumed biological underpinnings, social constructionists see emotions as socially and culturally shaped and malleable products that are strongly influenced by language-specific emotion words and emotional practices. Thus, emotion words do not seem to denote clear-cut psychological entities but themselves construct conceptual units for emotional events (Wilce, 2009:80). This turns emotions into more social and interpersonal phenomena that are embedded within the moral order of a given society or culture. For instance, the medieval emotion termed accidie - a feeling of tiredness and unwillingness to commit to one's religious 
duties - can only be understood within the social and historical context of religious practices in the middle ages and the corresponding moral order of Christianity (Harré and Finlay-Jones, 1986). Similarly, the Japanese emotion amae - a feeling of baby-like coquettish love towards and sweet dependence on the superior - seems natural in a Japanese cultural context but childish if performed by adults in the West (Morsbach and Tyler, 1986). From the perspective of relational work, these insights are particularly interesting as they show that emotions are not only recruited or referred to in social interaction, but that social interaction and the social and moral backgrounds in which it takes place itself shapes emotional interpretations in agreement with the social roles that are performed by the interactors.

While emotions are difficult to conceptualize, these complex syndrome categories must be claimed to function as important components of meaning. Since emotions serve the primary purpose of evaluating our inner and outer worlds of experience, we would like to define this emotional dimension of meaning as evaluative meaning. From a linguistic and discourse analytical perspective, however, it is important to emphasize that emotions are not empirically accessible on the basis of the methodological apparatuses available. Only external stimuli for emotional states and the expressive responses to those stimuli can be observed. For a linguistic analysis of emotions in the context of interpersonal pragmatics and relational work this entails that we cannot really refer to this fuzzy inner world of emotional reference, but that we can only analyze the range of multi-modal signals that are used by interactors to index potential emotional states (see also Wilce, 2009:10). For the analysis of relational work this means that emotions can only be analyzed as externalized and communicative phenomena rather than internal psychological states as we do not have empirical access to the latter. However, it is possible to engage with the presence of emotional cues or their striking absence when they would actually be expected according to the norms of the interaction in question and ask what these cues communicate as part of the relational work performed by the interactional partners. On the basis of these emotional signals, we must then infer the evaluative meaning that they might entail. Epistemologically speaking, the analysis of evaluative meaning through discourse analytical methods thus faces the very same problems of interpretation as any other inferential model of meaning generation. This intricate relationship between emotional signals and evaluative meanings will be further discussed in section 5. In what follows, we will first turn to the connections between emotions and interpersonal relationships.

\section{What are the links between emotions and interpersonal relationships?}

Human sociality cannot be understood without the positive and negative associations that we have with different social relationships. Interestingly, the connection between emotions and interpersonal relationships is highly reciprocal: emotions are decisive for the construal of the relationship and social relationships are the most important source for human emotions. In the psychological literature this link between emotional 
communication and sociality is best exemplified by the success of Goleman's book Emotional Intelligence (1995), which made it onto the bestseller lists with the title turning into a buzz word, especially in the corporate sector. While one may object to such a colloquialization and objectification of the yet underexplored connections between emotion and human intelligence, Goleman's book provides further evidence that emotional dimensions cannot be excluded from research on language use and the study of relational

work. In this section, we would therefore like to engage with the principles of emotion in social interaction as sketched in the area of communication studies. These principles constitute important theoretical guidelines for integrating emotional parameters into interpersonal pragmatics and relational work. Note that communication studies have also investigated the role of emotional communication in diverse communicative practices such as conflicts, family conversation, marriage counselling, etc. While these specific usage contexts are highly relevant with regard to relational work, we do not have the space to review them here (for an overview see Andersen and Guerrero, 1998a).

Andersen and Guerrero (1998b) discuss six principles that determine the links between emotional communication and the interactional management of interpersonal relationships:

1. "Socially adaptive emotional communication is positively selected in the evolutionary process." (50)

2. "Socialization processes guide how individuals manage their communication of emotion" (52)

3. "Interpersonal schemata [scripts of 'normal behaviour'], including goals, needs, desires, and expectations affect how and when emotion is experienced and communicated." (64)

4. "Interpersonal communication ${ }^{3}$ is the primary elicitor of most emotions." (57)

5. "An essential feature of the emotional experience is expression via interpersonal communication." (73)

6. "Emotions generate other emotions in interaction chains." (82)

In our interpretation, the first three principles can be read as foundational principles that determine the basic biological, socio-cultural, and cognitive connections between emotion and social interaction. The last three principles are focused on the actual role that emotions play in interpersonal communication and vice versa.

The first principle addresses the central function of emotions for human bonding and the management of interpersonal relationships. Human sociality is fundamentally grounded in our ability to empathize and emote with others. This is supported by both ontogenetic and phylogenetic studies on human social skills (Bråten, 2007; Malloch and Trevarthen, 2009; Turner, 2000, 2007; Turner and Stets, 2005). Emotions guide and influence the dynamic and interactive construal of relational meaning. On the one hand,

\footnotetext{
${ }^{3}$ Andersen and Guerrero (1998b) use the term "socially adaptive emotional communication" to refer to the evolutionary perspective of the phylogenesis of the human species, while "interpersonal communication" is synonymous to our concept of linguistic interaction.
} 
humans seem to have an innate "need for frequent, affectively pleasant interactions with a few other people in the context of a temporally stable and enduring framework of affective concern for each other's welfare" (Baumeister and Leary, 1995:497). This seems to start from the very beginning of human ontogeny because research in developmental psychology shows how distressed babies become when their caretakers are in their presence but do not produce any emotional signals for bonding with their babies (Tronick, 2005).

Beyond providing the fundamental glue for relating, emotions also play a decisive role for interactional practices of social sanctioning: "[O]ne of the basic functions of emotion is to regulate behaviour so as to form and maintain social bonds" (Baumeister and Leary, $1995: 497)$. Turner $(2000,2007)$ goes as far as to claim that development of a more subtle repertoire of emotions and emotional expressions in itself was the key for turning humans into the socially adaptive animals that we are expected to be these days (see also Planalp, 1999:137). Most importantly, Turner argues that humans had to considerably increase their repertoire of positive emotions and corresponding displays in order to establish and maintain close social bonds. This is also in agreement with Jing-Schmidt's (2007) cognitivelinguistic analysis of the negativity bias (Rozin and Royzman, 2001), which can be described as a threat bias that is linked to dangerous situations and calls for quick emotional responses. In line with this bias, Jing-Schmidt (2007:419) argues that the evolutionary more basic emotional tendency to focus on negative (social) information is an evolutionary advantage as it is an "automatic vigilance strategy". In contrast, the Polyanna effect (or positivity bias) refers to the general tendency to use evaluatively positive words more often than evaluatively negative ones. This means that humans try to overcome the negativity bias by trying to establish a positive orientation to their worlds of experience through symbolic practices (Jing-Schmidt, 2007:422). In the evolution of human sociality, the development of a repertoire of positive emotions as well as strategies for sanctioning and inhibiting the display of negative ones thus seems to play a key role for the central human ability to construct relationships through discursive practices. Therefore, a discursive-pragmatic model of interpersonal communication, which links human emotionality with interactional skills and practices, should also put the connection between relational work and its emotional component at the centre stage of a theory of human sociality and linguistic-communicative capacities.

This evolutionary idea is also encapsulated in Andersen and Guerrero's principle 2, which applies to the development of human individuals rather than the development of the human species. Human beings can only learn about appropriate social behaviour and the corresponding norms of emotional communication through complex and culturespecific processes of socialization in which acts of parental guidance play very important roles. Through social referencing, emotional displays support socialization and enculturation processes (Nishdia and Lillard, 2007). Emotional displays play Parents work as models of emotional display regarding the norms and moral codes of their cultural environments. In their various studies in the area of language socialization, Ochs 
and Schieffelin $(1984,1989)$ have also proposed that a child learns about the world of experience by paying attention to the linguistic expression of affect communicated by their parents (Ochs and Schieffelin, 1989:8). They revealed intricate and reciprocal connections between emotion, language learning and cultural learning (Ochs and Schieffelin, 1984; Ochs, 1986). Language socialization thus becomes "socialization both to and through the use of language" (Wilce, 2009:55). To account for the children's ability of learning about the world through their parents' emoting, these authors also developed a complex grid of emotional cues that may signal emotional orientations in and through language (see below).

Principle 2 is fully compatible with the social constructionist view of emotions as discursive constructs rather than purely internalized psychological states. Moreover, it ties in nicely with our idea that relational work (and emotional practices of social sanctioning) can only emerge relative to the background of social contexts and their corresponding norms. We have argued that these norms are stored as cognitive frames in the interactors' long-term memories. This cognitive dimension of relational work ties in with the third principle proposed by Andersen and Guerrero; interpersonal schemata as well as the interactors' goals and expectations work as an important benchmark for evaluating the communicative behaviour of the partner. We will engage with this principle in more detail in section 5 .

The last three principles address the connections between emotions and actual practices of interpersonal communication and social bonding. The fourth principle is of central importance for our bringing together relational work and emotion. The very fact that people work on their social positions through relational work is the primary cause for emotional experience: "[...] the primary elicitor of emotions is interpersonal interaction. [...]. Moreover, people often strategically induce emotional states in others as a way of achieving interpersonal goals" (Andersen and Guerrero, 1998b:64). Along these lines, social relationships and social interactions are the most important source for human emotions (Andersen and Guerrero, 1998b). While we may also love, hate, or fear specific objects or creatures, we live in socially constructed worlds of experience and primarily evaluate and make sense of them through the human agents with whom we interact and who influence our emotional states (see also Harré, 1986a,b).

In line with principle 5, interlocutors communicate their emotional orientations to the other for the strategic management of their relational goals (see also Frijda and Mesquita, 1994). The emotional orientation of the interactional partners towards both the symbolic construction of (process), as well as the emergent construct of their relationship (product) is decisive for relational work. As a result, relational work is often accompanied by an emotional interaction in accord with the sixth principle proposed by Andersen and Guerrero. But to be able to communicate emotionality to the interactional partner as well as to literally 'perceive' emotional states in the other, human beings depend on complex semiotic tools of emotional signalling. Remember that these emotional signals just constitute one component of the complex emotional syndromes but in order to understand how relational work is linked to emotions through complex practices of 
emotional signalling we will now scrutinize this semiotic dimension of emotions in the following section.

\section{How are emotions signalled interaction and how is the communication of emotions influenced by social and cultural norms?}

If we wish to understand the relational work performed by interactors, we centrally need to account for how they use emotional cues in order to express or communicate their evaluations of the current state-of-affairs including their relationship to their communicative partners (see Caffi and Janney, 1994). What becomes immediately apparent is that emotional cues are not only linguistic but multi-modal in nature. For this reason, it is necessary to adopt a multi-modal stance (for the importance of this stance, see, e.g. Jones, 2013; Norris, 2004; LeVine and Scollon, 2004; Kress and van Leeuwen, 1996) in order to understand better how relationships are created, challenged and maintained through complex signalling practices.

Research in communication studies has provided an overview of the repertoire of emotional cues that are recruited for interaction. For example, Planalp (1998) lists and discusses research on a range of vocal, verbal, body, physiological, and facial cues. This repertoire is presented in Table 2 . These emotional signals provide the basis for communicating and inferring evaluative meanings, i.e. the actual emotive states (e.g. love, embarrassment, shame, guilt) that we display or that we attribute to our partners. Among these cues, facial expressions constitute the most explored set of cues. In the tradition of Darwin's (1872) early studies on parallels between facial expressions between humans and animals, pioneering research was conducted on the basis of naturalistic observation (Tomkins, 1962, 1963; Izard, 1971). But most importantly, Ekman's experimental studies on the recognition of emotions expressed through the face paved the way for the search of basic and universal sets of emotions that are associated with specific configurations of rapid cues in the face (Ekman and Friesen, 1975). Ekman and Friesen (1978) developed the Facial Action Coding System to analyze the facial 


\begin{tabular}{ll}
\hline Class of cues & Forms of realization \\
\hline Vocal cues & Voice quality: low, loud, slow, fast, trembling, high-pitched, \\
monotonous, animate voice \\
Lerbal cues & Metaphors \\
& Speech acts \\
& Emotional discourse practices, e.g. therapeutic discourse \\
& Animated, energetic movement \\
& Physical actions: throwing things, making threatening movements, \\
Body cues & kissing, caressing \\
& Gait: walking heavily, lightly, arm swing, length/speed of stride \\
& Body posture: stiff/rigid, droopy, upright \\
& Hands/arms gestures: hand emblems, clenching hands or fists \\
& Blushing, pupil dilation, heart rate, breathing, skin temperature \\
Fhysiological cues & Facial expressions of emotions through forehead and eyebrows, eyes \\
Facial cues & and eyelids, and the lower face (mouth, lips, labionasal folds) \\
\hline
\end{tabular}

expression of the six basic emotions surprise, anger, fear, sadness, happiness, and disgust. In a series of experiments, Ekman and his colleagues tried to show that humans employ the face to distinctively encode these specific emotion categories (see, e.g. Ekman and Friesen, 1975). These claims have not remained uncontroversial, however. Especially evidence from anthropological studies questions both the existence of basic emotions as well as their assumed universality (e.g. Heelas, 1996; Michel et al., 2006). It is shown that informants judge facial expressions less accurately than suggested by Ekman. This might speak for a less isomorphic connection between emotional categories and facial expressions and promote the idea that emotional states are pointed to rather than encoded through various semiotic channels. In other words, emotional cues indicate emotional orientations rather than a distinct set of emotion categories. Nevertheless, in psychology, Ekman's programme has been so influential that facial emotional expression basically became associated with emotional expression as such. As a consequence, the other emotional cues received much less attention.

The interface between the verbal and the vocal cues for emotions has been more prominently explored in anthropological linguistics (see, e.g. Couper-Kuhlen, 2011). To account for the children's ability of learning about the world through their parents' emoting, Ochs and Schieffelin (1989) developed a complex grid of emotional cues that may signal emotional orientations in and through language. Their framework situates emotional signals on all levels of linguistic organization from minute vocal cues to complete discourse practices. A further substantial contribution by this study consists in a classification of the function, the semantic scope, and the distribution of affective signals. From the perspective of relational work, Ochs and Schieffelin's framework constitutes a comprehensive overview of the linguistic cues that interactors may recruit for symbolizing emotional orientations in their performance of relational work. (These dimensions will be 
further elaborated below). In line with the idea of social referencing, interactors may attend to these cues to learn about the other's evaluation of the actual social world of experience. However, it is important to emphasize that none of these cues encode a specific emotional category. Instead, they index emotional states. For instance, it is not always clear whether tears signal sadness or overwhelming happiness. When seeing the face of a crying sportsperson out of context, we cannot decide whether he/she is crying because of losing a race (pain/sadness) or winning an Olympic gold medal (happiness/satisfaction). In other words, the specific quality of these states has to be inferred from the cue (tears) as well as further non-verbal and contextual information.

From a more decidedly linguistic perspective, alternative frameworks for the analysis of vocal or verbal cues have been proposed by Foolen (1997), Fiehler (2002), Oster (2010), Schwarz-Friesel (2007) or Reber (2012). They also engage with the set of potential linguistic vehicles for the expression and description of emotion and mention emotion words (e.g. love, hate), word connotations (e.g. war, party), interjections (oh, ah, geez), metaphors (in seventh heaven, down in the dumps), optative sentences (I wish you were here), etc. While it is impossible to engage with these structures in detail here, it is important to emphasize that the importance of analysing the emotional components in language is increasingly being recognized in linguistics (see, e.g. Foolen, 2012). The problem with most of these linguistic approaches is that they limit emotional display to verbal communication while ignoring the interaction of the verbal messages with other emotional cues. From her stance in communication studies, this research lacuna is explicitly highlighted by Planalp:

Even though researchers tend to study amputated heads, decapitated bodies, disembodied and content-free voices, and decontextualized words, we know that in some way they go together. What is singularly lacking is any plausible account of how cues are combined into complex multichannel messages about emotion, much less accounts of how they are produced and interpreted. (Planalp, 1998:37)

In line with the central tenets of the study of relational work, we suggest that a discourseanalytical perspective on communicative signalling and sense-making allows us to bridge this gap as it allows a process-oriented analysis of emotional cueing in situated interactional practices. ${ }^{4}$

Indeed, more multi-modal analyses of emotional signals have been developed in the ethnography of communication, most importantly, in the work of Charles and Marjorie Goodwin. Promoting an embodied view of emotional signalling within "processes of interaction" (Goodwin and Goodwin, 2001:253; Goodwin et al., 2002), they adopt a highly

\footnotetext{
${ }^{4}$ Depending on your research question and your data situation, we agree with Spencer-Oatey (this volume), who studies workplace interaction and claims that interviews with participants might help in establishing emotional reactions by respondents in retrospect. This is especially the case if the display of emotions is withheld because the norms of the situation require this. In our paper, however, we wish to concentrate on what is observable and how to best grasp it with analytical tools. In addition, we argue that the absence of a marked emotional stance in itself can also have signalling force. We elaborate on this in the continuation of section 5 and in the conclusion.
} 
dynamic and multi-modal view of emotional communication, suggesting that affective stance in relational work cannot be understood from a purely language-centric perspective on semiotic behaviour. This strongly supports a more comprehensive discursive-pragmatic approach to relational work and its emotional component that embraces multi-modality.

"Embodied intersubjective participation" (Wilce, 2009:51) is also the focus of Matoesian's (2005) close conversation-analytical reading of a focus-group interview in the context of a US training programme in community policing. Matoesian analyses how the interactors signal their stance towards the propositions made during the interview. But rather than regarding stance marking as a purely speaker-centred linguistic affair, Matoesian shows that

[...] stance functions not only through grammar prosody and paralinguistic features, nor even only through the autonomous body; it also emerges interactively and incrementally in the embodied multimodal projection and negotiation of participation frameworks through sequentially organized rhythms of language and the body. (Matoesian, 2005:169)

From the perspective of relational work this is a striking insight, as the "emotionally charged recipient activity" (Matoesian, 2005:182) of the communicative partners does not only pare down to a matter of expressing internal bodily states and their appraisal but rather points to the joint and strategic management of emotional orientations within embodied interactional practices that integrate a variety of cues.

As stated above, the analysis of emotionality on the basis of emotional cues alone is highly complicated for two reasons. First, emotional cues are often withheld or modulated because of social and cultural norms of display. Second, there is no direct visible link between emotional cues and internal emotional states. Rather, from an interactional perspective emotional orientations must primarily be seen as semiotic constructs that help the communicative partners making their evaluations of a given state-of-affairs socially accessible and thus socially accountable. While some cues may result from spontaneous expressions of genuine emotional states, other cues may be used strategically to emotionalize a given message in the absence of actual arousal. Degrees of control on this continuum from spontaneous to strategic emotional display are very difficult, if not impossible, to distinguish from an interaction-analytical perspective. However, we would like to claim that this is not of central relevance for an analysis of emotional signalling from this empirical vantage point. What counts as objects of analysis within an interactionanalytical framework are the recognizable emotional displays as well as the interactional partners' orientations to these displays in their communicative engagements. In this the analysts do not differ from the interlocutors as both have to rely on perceived cues in order to arrive at assumptions about intentions and emotional stance. It is worthwhile to point out that not every individual is equally adept in reading and interpreting emotional cues and that social norms are negotiated in a discursive process. This and the fact that emotional cues are often ambiguous and only clarified in the development of interaction 
explains that often partners misread and misinterpret emotional signalling, and that different people may react differently to the same emotional displays.

The dimension of emotion management through communication has been addressed in communication studies. Actually, emotion management within relational episodes is a highly familiar phenomenon. If we did not adapt our emotions and their display to others, we would not feel torn between expressing what we really feel and expressing what the other might wish us to express. Rather, we adapt our emotions and their expression to our audience in order to modulate social connections. Following Hochschild (1979), Planalp (1999:75-76) distinguishes two main strategies for emotion management in stage acting and then expands these concepts to communication in general. On the one hand, surface acting relates to the management of emotional expressions. This corresponds to the application of a number of display rules that were proposed by Ekman and Friesen (1975) with regard to the communicative management of facial expressions. By applying the display rules, communicators may change the expressive surface of their emotional states by simulating, inhibiting, intensifying, de-intensifying, or masking their actual emotions in order to follow culture-specific norms of appropriate emotional display. On the other hand, deep acting involves attempts to actually change one's

internal emotional states. We can do so by avoiding emotion-triggering situations, by influencing our appraisals of such situations, by manipulating our physiological reactions (e.g. by taking drugs), or by changing our action tendencies.

The idea of emotion management is of central importance for the concept of relational work. If we can strategically manipulate our emotional orientations to influence our relationships with our interactional partners either through surface acting or deep acting, then interlocutors must be expected to pay close attention to the presence or absence of emotional signals for making sense of their actual social position relative to the other. This said, it is important to emphasize that it is impossible to investigate processes of surface acting or deep acting on the basis of a close reading of interactional data only. As we do not know about the interactors' internal emotional states, we also do not know whether the emotions indexed through the multi-modal cues are 'authentic' or not. However, as argued above, this does not constitute a central problem for the impact of emotions on relational work as a clear distinction between the spontaneous expression and the strategic communication of emotional stances is basically impossible to draw as emotions and their displays are so deeply socialized (Planalp, 1998:31-32).

The relational work involved in negotiating a relationship is fundamentally linked to the active management of evaluations in and through communication. As this emotional communication is subject to social and cultural norms, we can address violations of corresponding display rules as a final dimension for integrating emotions and relational work. Our performance of specific social roles is associated with social normative expectations of appropriate emotional states and behaviours (Hochschild, 1983; Planalp, 1999:93--96; Culpeper, 2011; Spencer-Oatey, 2011). Emotions seem to play a role when satisfactions or violations of frame-based expectations and personal preferences occur. For instance, violations may trigger negative emotions, which will then be part and parcel 
of judgments about relational work. These situated judgements present the link to politeness research and to work on identity construction (cf. Locher, 2008, 2012). In Locher and Langlotz (2008), we argued that emotions play a crucial role in the creation and interpretation of these judgments. For example, positive emotions are triggered when being treated politely, or negative emotions when treated rudely. A recent and interesting socio-cognitive model for the role of emotions in the interpretation of offence and impoliteness is offered by Culpeper (2011:65-70). Most importantly, he also assumes a close link between breaching social expectations and norms, emotional reactions and cognitive processes of appraisal. His approach is thus largely compatible with our own ideas on the link between relational work and emotions.

Considering these insights as a challenge for developing a more comprehensive approach to relational work, we will now chart possible routes to account for multi-modal practices of emotional signalling as well as the construction of emotional meaning within this particular framework.

\section{Calvin and Susie at relational work}

We would like to sketch our own approach to this issue by engaging with the following cartoon. It captures the beginning of a relational episode between Calvin, one of the two main protagonists from Bill Watterson's fictive 'Calvin and Hobbes' cartoon world, and Susie, his less than favourite class mate. The brief interaction takes place in the context of an American school (note the lockers in the background) with Calvin and Susie having been assigned to work together on a report (Fig. 1).

We are very much aware of the fact that this piece of data is no substitute for the complexity of recorded face-to-face data of naturally occurring interaction between flesh and blood participants. However, the cartoon is also naturally
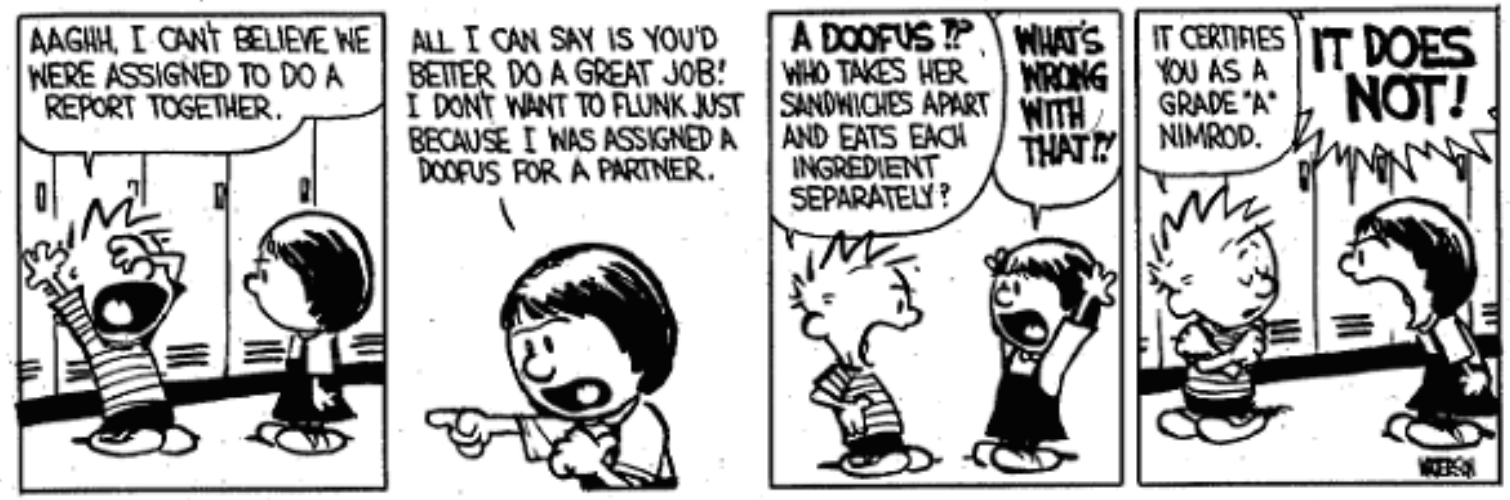

Fig. 1. The Authoritative Calvin and Hobbes, pp. 121-124. (CALVIN AND HOBBES (c) Watterson. Used by permission of Universal Uclick. All rights reserved.)

occurring in the sense that this data was neither elicited with a particular research question in mind nor produced for linguistic analysis as such. We realize that comic strips and graphic novels constitute a genre in its own right (cf. Saraceni, 2003; Kimmich, 2008; 
Adler, 2011), and consider the example suitable for an analysis of the relational and emotional dimensions of interaction. This is the case especially because we are presented with an exaggerated and condensed representation of the combination of multi-modal relational cues. Following Alvarez-Pereyre (2011), who assesses the usefulness of telecinematic data for linguistic analysis, we make an analogous case for data from cartoons and argue with Alvarez-Pereyre (2011) that

The very fact that the lexicogrammatical structures have been, carefully and nonspontaneously, chosen to fulfill the particular functions assigned, makes them extremely good specimens for the study of the relationships between forms, meanings and functions. (Alvarez-Pereyre, 2011:62, emphasis in original)

The target audience being adults who can appreciate the wisdom of the characters' observations of the daily life of growing- up in the United States and who can grasp the irony and humour entailed in the episodes, draw on their cultural knowledge of the described scenes and include the emotional multi-modal cues in their interpretation of the cartoon's message. As outside observers, the readers may appreciate the incongruity between Calvin's and Susie's rather direct and uninhibited emotional signalling in the context of school and the social norms of emotional signalling in an adult workplace context in which two people have been assigned to collaborate as a team. We assume that in the world of adults in a comparable cultural (Anglo Western) context, it would be considered highly inappropriate to express one's emotional stance towards the collaborator in such an unfiltered way. Knowing about these norms and their violation depicted in the cartoon, we can thus laugh at Calvin's and Susie's exaggerated display of their contempt towards each other. Although fictional, this short interactional sequence is thus very interesting for analysing links between emotion and relational work. More specifically, we would like to argue that the development of Calvin and Susie's relationship and our humorous appreciation of it cannot be understood, if the evaluative components and their situated interpretation are disregarded. In what follows, we will offer our own understanding and interpretation of this short sequence, being well aware that readers from different cultural backgrounds or with different emotional sensitivities might evaluate the emotional cues we will identify differently. Having said this, our purpose is to show a potential reading in order to demonstrate how a combination of emotional and relational cues influence each other in a dynamic process of sense-making.

It is crucial to remember that in section 3 we have defined emotions as complex and dynamic processes. However, only their (communicative) stimuli and communicative expressions become accessible to the interactors and the discourse analyst. Along these lines, verbal cues such as Aaghh, I can't believe, doofus, nimrod as well as Calvin and Susie's body positions, gestures, and facial expressions signal emotions in the cartoon. The cues frame their emotional states and allow them to express and symbolize their annoyance. Emotional display thus works as a powerful control mechanism that provides an evaluative framing for both the social relationship between the school kids and the contents of their 
transaction. Thus, the protagonists' relational work (and, of course, the readers' reception and interpretation of what happens 'between' the boy and the girl) involves complex and subtle semiotic processes of positive and negative emotional signalling which provide the basis for inferring corresponding evaluations. Table 3 summarizes the verbal features and systematizes them relative to different levels of linguistic analysis in accord with Ochs and Schiefflin's (1989) classification of affect cues (see above).

Table 3. Ochs' and Schieffelin's (1989) classificatory grid, abbreviated for the linguistic expression of affect relevant to our data set.

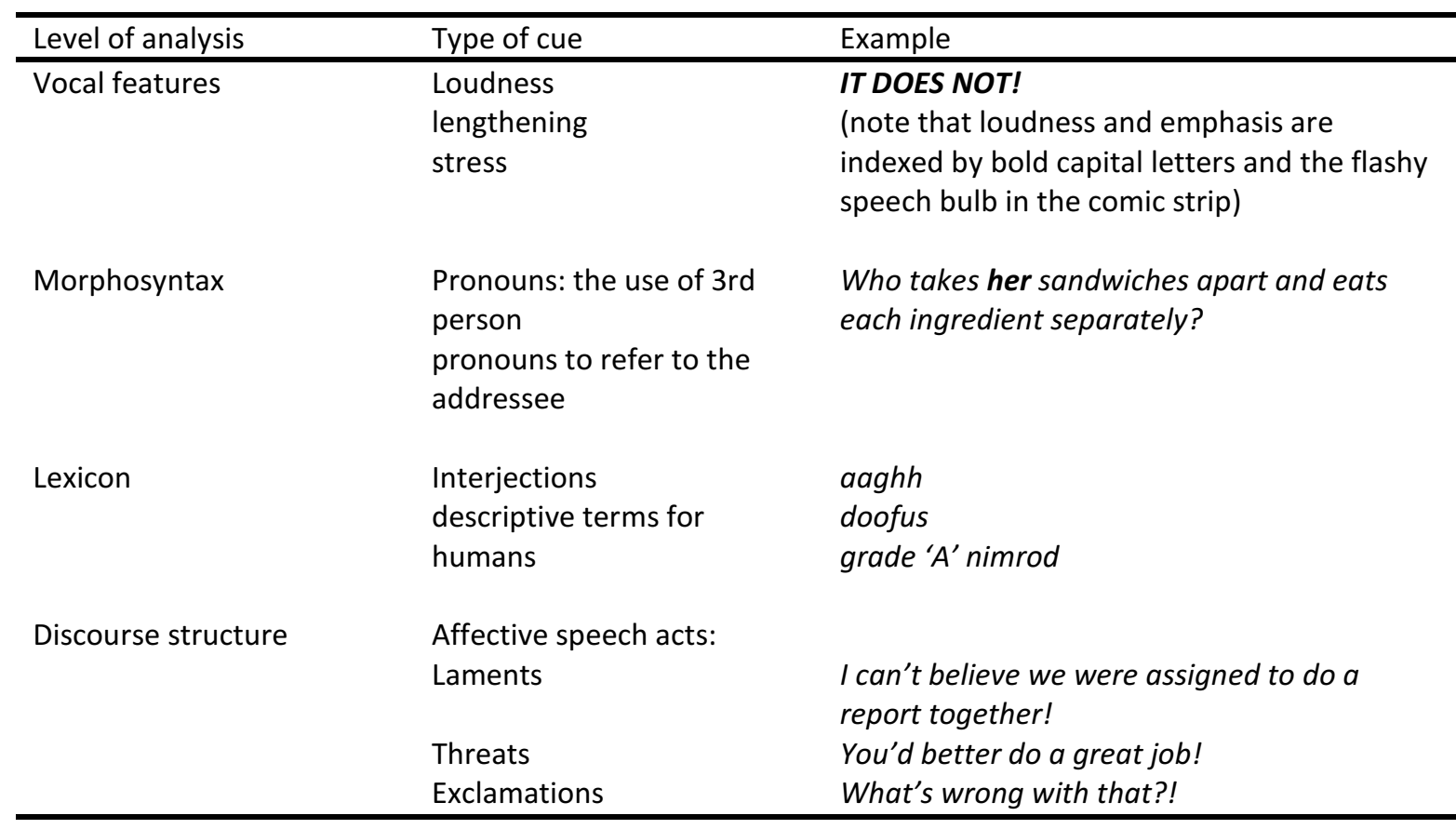

In line with Ochs and Schieffelin's framework we can further analyze the function, the semantic scope, and the distribution of these affective signals. First, the authors distinguish between affect specifiers and affect intensifiers (Ochs and Schieffelin, 1989:1415). While the former specify the emotional orientation of an utterance, the latter rather modulate its affective intensity. Thus, Calvin's interjection Aaghh can be seen as an affect intensifier, whereas I can't believe specifies Calvin's exasperation. Concerning the scope of such cues, a distinction is made between signals that evaluate a given referent (e.g. doofus for Calvin), cues that evaluate a full proposition (e.g. I can't believe emotionally frames the proposition we were assigned to do a report together), and cues that are linked to a sequence of propositions (Ochs and Schieffelin, 1989:15-18). Finally, affective signals can appear in three positions relative to an utterance. Initiators, such as Aaghh, I can't believe, occur at the beginning and cast an emotional shadow onto the subsequent proposition. Concurrents provide emotional information along with the statement that is being made as in WHAT'S WRONG WITH THAT?! Here Susie raises her voice while making her point. Finally, terminators may be placed at the end of an utterance to provide an emotional evaluation after a given proposition is made. To provide an example, we can modify Calvin's initial statement into We were assigned to do a report together, poor me! 
These emotional signals play a decisive role for the construal of the relationship. The relational work between Calvin and Susie thus involves a complex interplay between three dimensions. By scrutinizing the first panel, these dimensions can be outlined as follows:

a. The construal of conceptual content: When Calvin states we were assigned to do a report together, he asserts a fact that changes his world of social experience. Doing a report together implies teamwork and the formation of a relationship.

b. The construal of the relationship: Calvin's undesired relationship to Susie stands at the centre of his assertion. To highlight the emerging, novel social constellation, we is used as a person deictic that points to the two interactors. The discursive negotiation of the interactors' self-images relative to their unwilling relationship is the main theme of the subsequent panels.

c. Calvin's emotional evaluation of a. and b.: Calvin's emotional evaluation, i.e. his exasperation is shown in a highly multi-modal form involving his hand gestures, the reclined body position, the sigh Aaghh as well as the expression I can't believe. In the first panel, the negative evaluation of the assertion we were assigned to do a report together is primarily evoked and mediated through these signals.

While this list seems to suggest that these three dimensions of signalling and sense-making are separable, we prefer to argue that they stand in a reciprocal relationship, which connects them inextricably. Although some signals such as the personal pronoun we or the interjection aaghh seem to be specialized for the coordination of relational and evaluative meaning, respectively, the whole utterance and the accompanying non-verbal cues merge into a complex composite signal (Clark, 1996:178-179, see below). In addition, we can see that the interactional negotiation of these evaluations is managed through a discursive process of co-constructing this relational meaning by mutually orienting to their appraisals of the assignment-task, their emotional perspective on future collaboration, as well as their evaluation of each other.

It is important to note that from a linguistic perspective it is not relevant whether Calvin is truly annoyed and exasperated. Of course, Calvin does not exist beyond the interesting cluster of lines that was created by Watterson. Hence, genuine emotions are not involved in our example. However, the comic strip manages to create the fictional illusion of emotionality at work. As readers we interpret Calvin's emotional signals as expressing his emotional stance to Susie to influence her strategically and to let her know that she cannot expect too much cooperation from him. With regard to Calvin's performance within the world of the cartoon this would mean that Calvin may indeed spontaneously feel exasperated. But through socialization he would also have learned how to intersubjectively signal and to construct this emotional state for its recognition by Susie. The communicative manipulation of Susie's attention in line with the idea of exasperation therefore cannot be understood without reference to non-spontaneous, culture-specific norms of emotional display. It is these norms that we can rely on as readers to attribute evaluative meaning to Calvin's utterance in the first panel.

From the initial analysis of this comic strip we can therefore distill a number of key insights with regard to the connection between relational work and emotion as illustrated 
in Fig. 2. The relationship is the product that emerges from the interplay of the following simultaneous dimensions:

- First, we have claimed that relationships are social constructs that emerge dynamically through joint practices that contain emotional components (A).

- In the cartoon, we must thus scrutinize the dynamic activation of multi-modal repertoire of vocal, verbal, bodily, and facial cues (B).

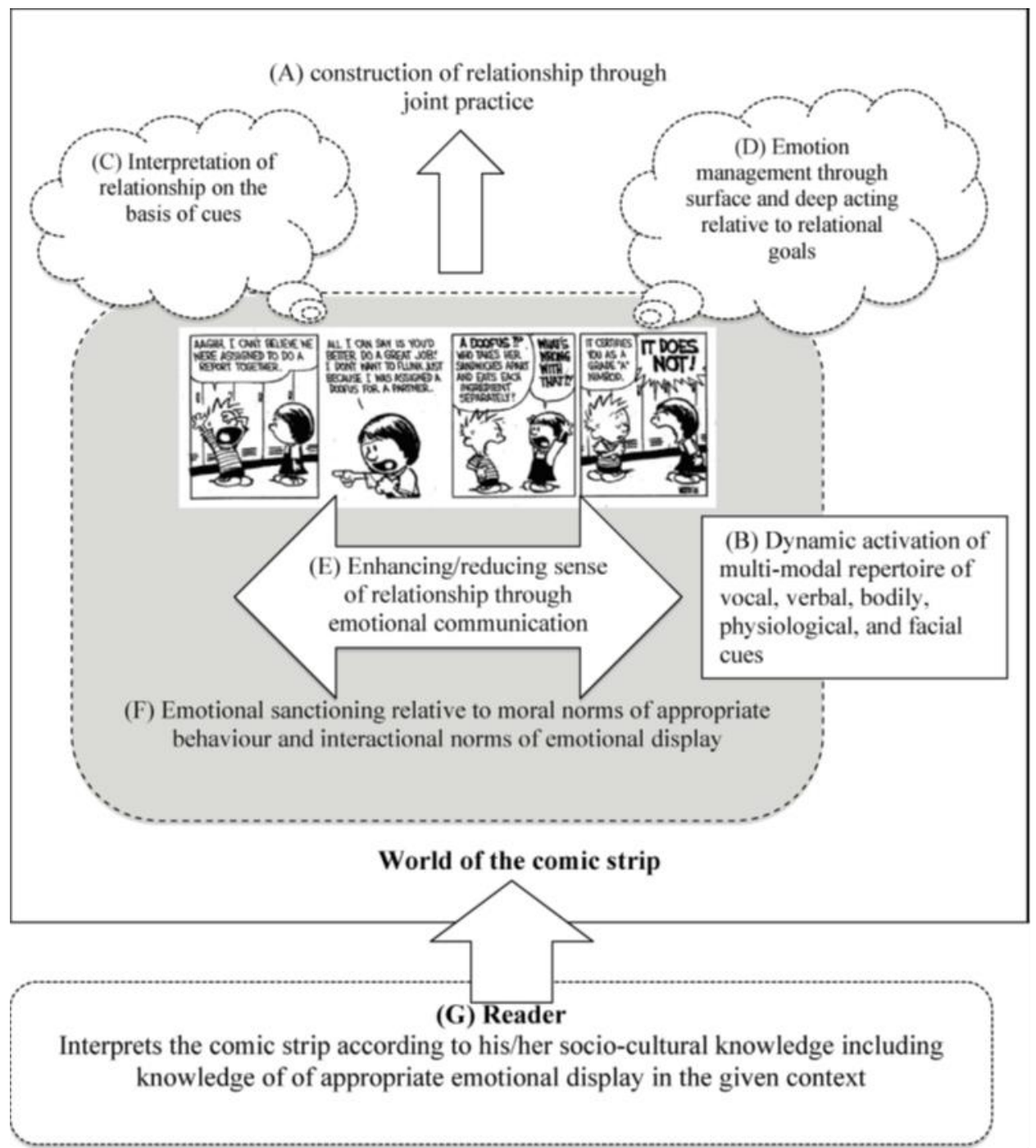

Fig. 2. Emotional components in relational work in the comic strip and their interpretation by the reader.

- On the cognitive plane, the multi-modal signalling strategies guide and shape joint and intersubjective processes of inferring relational meaning (C).

- In this process, the interactional partners manage their emotional displays relative to their relational goals (D).

- Emotional orientations thus emerge as semiotic constructs. This emotional communication results in enhancing, maintaining or reducing the sense of relationship between the interactional partners $(E)$.

- This process of emotional sanctioning works relative to moral norms of appropriate behaviour and interactional norms of emotional display (F). 
- The fictional display of Calvin and Susie's emotional signals is interpreted by the reader who must activate his/her socio-cultural knowledge of emotional display in school and other contexts in order to interpret the relational work performed in the cartoon $(G)$.

In the next section we want to explore these topics in further detail. By offering our own interpretation of the brief interaction between Calvin and Susie, we therefore move from these theoretical considerations to the more practical question of how emotional signals can be analyzed in this concrete instance of relational work.

\section{Relationship construction through multi-modal emotional display}

In this section we will address the challenge of handling the discursive management of relationship evaluation through multi-modal and composite emotional signals in interaction. In doing so we will make use of Clark's seminal work on Using Language from 1996, more specifically his semiotic model of communicative signalling. He proposes that interactors do not merely encode meanings into symbolic units but rather engage in complex processes of multimodal signalling for describing, indicating, and demonstrating their stances to one another. Describing is seen as the method of signalling that is based on symbolic convention -- centrally but not exclusively it involves linguistic communication (e.g. I am sad). Indicating is used to point to a contiguous object or referent (e.g. turning away from the communicative partner to index annoyance). Demonstrating is a form of imitating a phenomenon (e.g. reproducing the surprised facial expression of another person during a story-telling event).

Moreover, Clark highlights that the creation of meaning is achieved by joint actions in which the speaker and the listener simultaneously play active roles on four different levels: (1) executing behaviours and attending to them, (2) presenting signals and identifying them, (3) signalling meanings and recognizing them, and (4) proposing a joint project and considering this joint project. Thus, signalling is a dynamic process that requires coordination and fine-tuning between the partners in a conversation.

We agree with Clark that communicative actions must be joint actions in which both the speaker and the listener play an active role. For the purpose of our analysis, we will not be able to present the finer details of Clark's theory here. However, following Clark, we assume that emotional signals have a complex, composite structure, which emerges in dynamic processes of emotional signalling that are displayed for reception by the interactional partner(s) (Planalp and Knie, 2002). In an actual conversation, speakers beyond producing their own emotional displays are also reacting to the emotional reactions of the recipients while 'formulating'. At the same time, listeners are both reacting to and interpreting the speaker's emotional signals to evaluate what is being said consciously as well as unconsciously. ${ }^{5}$ Note that these displays are complex signals that

\footnotetext{
5 This is in line with the very recent Geneva Multimodal Emotion Portrayals (GEMEP) corpus that was developed in the context of the NCCR Affective Sciences: Emotion in Individual Behaviour and Social Processes
} 
are assembled from the repertoire of verbal and non-verbal cues that were presented in Tables 2 and 3. On the basis of these cues, the communicators can signal evaluations to the recipient and invite him/her to engage with the proposed appraisal. In other words, acts of creating evaluations involve both the signalling of these evaluations through emotional cues and their potential take-up. In what follows, we will first discuss the multimodal nature of emotional signalling and then discuss the complex process of creating evaluative meanings through the dynamically evoked composite signals.

\subsection{Multi-modal acts of emotional signalling}

Clark's view of signalling questions language-centred models of communication (Clark, 1996:Ch. 6). He rejects the idea that communicative signals are primarily 'linguistic' objects. By integrating other communicative modalities, Clark instead claims that signals are composite signals that are constructed by linguistic and non-linguistic methods of signalling. Thus, non-linguistic methods of signalling are not considered crude and unsystematic but part and parcel of communicative acts.

With reference to Peirce's (1977) theory of signs, Clark develops a dynamic theory of signalling. He does not regard signs as static units that are merely recruited as vehicles for encoding meaning. Instead,

[...] signaling is a mixture of three methods--describing-as, indicating and demonstrating. Describing-as is the only method that uses symbols, and it never works alone. In conversation, indicating is always required too. Of the three methods, demonstrating is the most neglected, yet is essential to everything from quotations to intonation to iconic gestures. (Clark, 1996:188)

Through the three methods of signalling composite signals are created online and designed for the identification by the recipient (Clark, 1996:178-179). By definition, composite signals are multi-modal, i.e. they recruit several instruments by means of which the communicators can 'manipulate' the semiotic landscape (Table 4).

Table 4. Methods and instruments of signalling.

\begin{tabular}{llll}
\hline & \multicolumn{2}{l}{ Method of signalling } & \\
\cline { 2 - 4 } Instrument & Describing-as & Indicating & Demonstrating \\
\hline Voice & & \\
Hands & & \\
Arms & & \\
Body & COMPOSITE SIGNAL & \\
Face & & \\
Eyes & & \\
\hline Adaptedfrom Clat & & \\
\hline
\end{tabular}

Adapted from Clark (1996).

(Bänziger and Scherer, 2010) and includes multimodal performances of emotions. This corpus is based on actor portrayals and is designed for experimental research. 

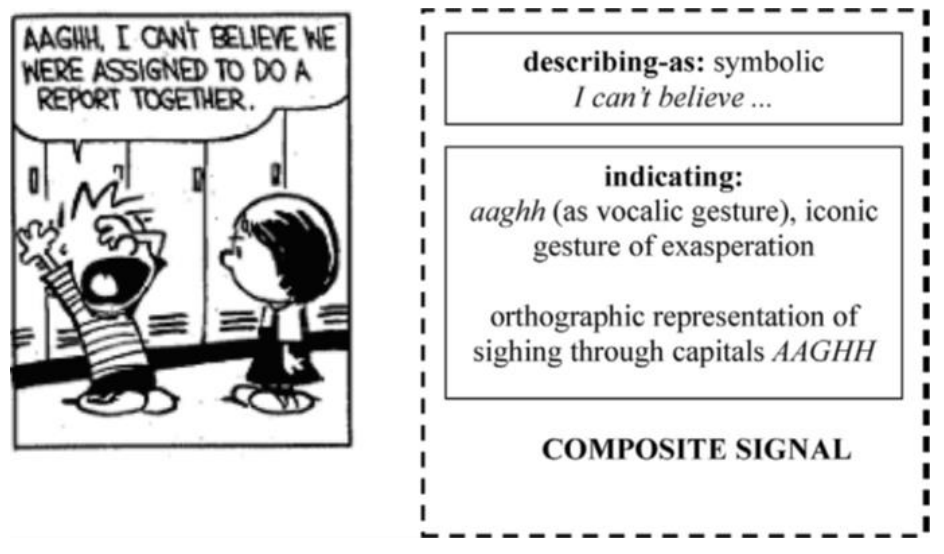

Fig. 3. Composite emotional signal.

The composite nature of communicative signals can again be illustrated by means of the first panel of our comic strip. Calvin's interjection Aaghh can be seen as a form of indicating. Note that interjections have been treated controversially in linguistics (for an overview see Schwarz-Friesel, 2007:157). Interjections like Aaghh do not have a conventionalized symbolic meaning. Here the lengthened production of the vocalic sound indexes Calvin's inner state of exasperation. On the other hand, interjections are usually positioned like sentence adverbs. Calvin's Aaghh is left-peripheral and therefore works as an initiator that evaluates the subsequent propositions through its negative emotional evaluation. Of course, in the strip this indexicality is added by the orthographic representation of the vocalic element. However, the reader is assumed to interpolate that in the world of the comic strip Calvin produces a vocal emotional cue. His use of I can't believe, however, is a symbolic act of describing his emotional state. Finally, he uses hand gestures and a facial expression of exasperation and is thus indicating his emotional stance through the body. Calvin's behaviours combine into a composite signal that, crucially, is designed for his addressee, Susie (Fig. 3). We can look at the entire stretch of interaction and analyze the three ways of signalling, describing-as, indicating and demonstrating with respect to how they are transmitted. This transmission is not achieved by language (or voice) only, but by the full repertoire of emotional cues sketched above. Let us first point out a couple of examples related to the voice as an instrument. This involves both vocal and verbal cues. In the category 'describing as', we find the exclamation I can't believe (panel 1), name calling doofus (panel 2 and 3), grade " $A$ " nimrod (panel 4), and word connotations flunk (panel 2). In the category indicating, we observe sighing Aaghh (panel 1). We also see the surprised/shocked, exasperated intonations for Calvin's A DOOFUS?? and Susie's WHAT'S WRONG WITH THAT?! that are indexed by?? and?!, respectively in panel 3. Moreover, there is shouting as in IT DOES NOT! In the graphic format of the comics, this is indicated by bigger capital letters in bold and the flashy speech bubble.

When turning to the analysis of the hands, arms and body posture as instruments for bodily gestures, we can highlight the following. In the category indicating, we can observe Susie's pointing finger as a bodily indicator of the direction of her verbal attack against Calvin in panel 2. We also see how Calvin reacts to Susie's attack when adopting a self- 
confident pose in panel 3 by placing his hands on his hips. Moreover, in panel 4, Calvin crosses his arms and turns away his body to indicate a defensive position against Susie's outraged exclamation. With regard to body posture Calvin's backward leaning body in panels 1 and 3 signal flight and reclining away from Susie, whereas Susie's forward leaning in panels 2

Table 5. Calvin's and Susie's composite emotional signals in panel 4.

\begin{tabular}{|c|c|c|c|c|}
\hline \multirow{3}{*}{ Instrument } & \multicolumn{4}{|c|}{ Method of signalling } \\
\hline & \multicolumn{2}{|l|}{ Describing-as } & \multicolumn{2}{|l|}{ Indicating } \\
\hline & Calvin & Susie & Calvin & Susie \\
\hline \multirow[t]{2}{*}{ Voice letters } & Grade "A" & & & Use of capitals and flashy speech \\
\hline & nimrod & & & bubble for shouting \\
\hline \multicolumn{5}{|l|}{$\operatorname{Hand}(\mathrm{s})$} \\
\hline $\operatorname{Arm}(\mathrm{s})$ & & & Crossed & Pulled down \\
\hline \multirow[t]{2}{*}{ Body } & & & Turned away from & Leaning forward \\
\hline & & & Susie & \\
\hline \multirow[t]{4}{*}{ Face } & & & Eyes closed & Hard stare \\
\hline & & & & Piercing eyes \\
\hline & & & & Eyebrows pulled down and \\
\hline & & & & together \\
\hline
\end{tabular}

and 4 underlines her attacks. We further see Calvin's and Susie's gestures of exasperation and/or desperation in panels 1 and 3, respectively. Both of them raise their arms upwards to convey their emotional agitation. ${ }^{6}$

Concerning the face and eyes we can point out that Calvin refuses eye contact in the first and fourth panel. This can be interpreted as indicating his negative evaluation of her. In panels 3 and 4 Calvin and Susie point to their anger through the face by their hard stares, piercing eyes and by pulling their eyebrows down and together (see Ekman and Friesen, 1975:97).

In the flow of the interaction, the different channels for emotional expression operate in conjunction for the display of composite emotional signals. The composite signals for panel 4 are summarized in Table 5. Calvin describes Susie as a grade " $A$ " nimrod. This creative compound undermines the positive connotations of the highest school grade by adding it as a premodifier to the head nimrod. In other words, nimrod can be interpreted as a terminator that casts a highly negative appraisal on Susie and her superior status within the world of the school. Calvin's verbal act is accompanied by his arm and body position. He turns away from Susie and crosses his arms. Moreover, he closes his eyes. In combination these signals iconically show how Calvin attempts to withdraw from her physically. This act is again countered by a composite emotional signal by Susie. Her anger is indicated in her facial expression and by her shouting IT DOES NOT! (Again, note that

\footnotetext{
${ }^{6}$ We interpret these gestures as indexes. Depending on their degree of conventionality, they could also be classified as symbolic gestures for exasperation and thus work as acts of describing-as. This shows that the distinction between conventional and spontaneous bodily signals for emotions is very difficult to draw.
} 
shouting is indexed through the comic strip convention of capitalizing the letters; Saraceni, 2003:20). These displays of anger are further supported by the arm and body position. Her pulled down arms and her forward leaning posture indicate her emotional attack against Calvin. (Since neither Calvin nor Susie explicitly quote/imitate the emotional signals by another person, we cannot find any instances of demonstrating in the short sequence.) These multi-modal acts of signalling manage emotional evaluations and thus influence the potential interpretation and take-up of the semiotic construction of Calvin and Susie's relationship. Our interpretation of this construction of evaluative meanings relative to the composite emotional signals in the four panels is offered in the next section.

\subsection{The dynamic construal of relationship evaluations}

The different emotional cues are combined into complex arrangements of composite signals. They reflect the mutually adaptive and intersubjective coordination of the interactors' emotional orientations. Through these semiotic tools they can dynamically 'manipulate' the emotion-related semiotic environment for apprehension, comprehension, and take-up by the interactional partners. We can thus use Calvin and Susie's actions as an analytical starting point to illustrate how relational work is supported by complex semiotic acts of displaying emotions. We will further address some challenges for more extensive empirical work on the basis of naturally occurring data at the end of this paper. In a nutshell, we see acts of emotional signalling realized in different ways. In section 6 we have argued that the creation of meaning for the construction of social orientation is based on a complex reciprocal relationship between conceptual content, relational meaning, and emotional evaluation.

So let us scrutinize the dynamic construal of Calvin's evaluation of their interpersonal relationship in panel 1. As we have argued, Calvin shows us an emotional evaluation by uttering: Aaghh I can't believe. Working as an initiator in the sense proposed by Ochs and Schieffelin (1989), these verbal cues provide an emotional contextualisation that place the rest of the message, i.e. the propositional content we were assigned to do a report together, under the given affective scope. Moreover, Aaghh functions as an affect intensifier and I can't believe specifies Calvin's state of exasperation. Calvin's emotional orientation is further supported by the non-verbal dimensions of Calvin's composite signal. The raised arm further intensifies his emotional agitation. The hand gesture of covering his eyes to closing his view from Susie as well as his reclined body posture signal his aversive appraisal of her as a project partner. Along these lines, Calvin provides a negative evaluation of both the assignment and the work relationship, which is indicated by the we, at the very beginning of the interaction. Susie takes this pessimistic framing of their joint future engagement up in the next panel.

In reaction to Calvin's stance, Susie produces an act that also combines conceptual content, relational meaning, and emotional evaluation: All I can say is you'd better do a great job! This is a bossy threat that places Susie in a superior and commanding position. The threat is underlined non-verbally by Susie's pointing finger and her forward leaning 
body position. The threat is followed by an account: I don't want to flunk just because I was assigned a doofus for a partner. Here, Susie distances herself emotionally from the joint venture and Calvin. Semiotically, this negative appraisal is signalled by the negative word connotation of the verb flunk, which evokes the school frame, and the derogatory name doofus. This word combines social and emotional meaning in a synthetic way. By calling Calvin a doofus she appraises his intellectual capacities in highly negative terms and thus positions herself as in an intellectually superior position. Moreover, she distances herself from Calvin by not addressing him directly, but by talking about a partner. Thus, while she attacks Calvin on a non-verbal level by her forward body she simultaneously increases the symbolic distance to him by uttering her non-flattering comparison.

In the third panel, Calvin takes up Susie's proposal for defining their relationship by expressing his shocked surprise and disbelief A DOOFUS?? The intensity of this appraisal is marked by the bold capital letters. Adopting a self-confident body position and putting on an angry face, Calvin himself produces a counter-argument to Susie's impertinent act of social categorization: Who takes her sandwiches apart and eats each ingredient separately? Thus, Calvin produces a negative evaluation of Susie's social image and sanctions her previous act of social positioning by pointing to her unconventional eating behaviours. Instead of characterizing her as intellectually superior, the image of Susie disassembling her sandwich into its atomic parts conceptualizes her as a nit-picking control freak with analytical capacities but no sense for enjoying the sensory pleasures of life. Moreover, rather than highlighting her intellectual superiority, it points to Susie's practical deficiencies. It is important to note that Calvin uses the third person pronoun her as a person deictic to distance himself from his addressee. Susie's reaction to this is an emotional re-evaluation of Calvin's comment that questions the supposed non-conformity of her behaviour. To save her social image, she exclaims: WHAT'S WRONG WITH THAT?! The raised voice and arms display the emotional arousal triggered by Calvin's symbolic sanctioning of her habits. She seems to be both in despair and scandalized by his act of retribution. This is also performed through her body posture, which reclines back and away from Calvin.

In the fourth panel, Calvin adds further fuel to the fire. His retort It certifies you as a grade " $A$ " nimrod nicely takes-up Susie's act of social categorization through derogatory name calling, but blends this symbolic gesture with the school-discourse of grading and certification (it certifies you as a ...). The compound grade " $A$ " nimrod undermines Susie's superior status within the world of the school. This act again triggers an angry contradiction by Susie (IT DOES NOT!) that sanctions Calvin's act of negative social categorization. In order to conclude our discussion, we will bring the different threads of reasoning together in our final section. 


\section{Conclusions: modelling emotional contributions in relational work - challenges for future research}

This paper took the research gap on the role of emotions within (im)politeness research as a starting point to review the existing literature on emotions in an endeavour to make the research insights usable for interpersonal pragmatics. Next to reviewing what the literature had to say on the nature of emotions, we also asked

- How are emotions signalled in interaction and how is the communication of emotions influenced by social and cultural norms?

- What are the links between emotions and interpersonal relationships?

In our analysis we see three main instances of clashes of expectations in connection with norms that are evident in Susie's and Calvin's interaction -- two inside and one outside the fictional world of the comic strip. The first one, at the very beginning, occurs when Calvin expresses his dissatisfaction with Susie as a partner for the school assignment. This implies that he has an ideal conceptualisation of a project partner -- and Susie does not fit. Susie in turn implies that Calvin jeopardizes her success in the project and is thus also less than ideal as a partner. The second instance of clash of expectations is centred on 'ways of eating a sandwich'. Calvin exposes Susie's habits as not confirming to his expectations of a norm and evaluates her social image negatively. She takes the bait and defends herself by challenging

his implicit norms. The last break of expectation occurs between the two kids' rather

explicit negative emotional display which runs against the adult norms of face-maintaining behaviour in accord with emotional display rules. It is important to point out that these interpretations rely on knowledge of interaction within a particular cultural context -- in this case US Western norms. These norms are not universally shared nor does our pointing out of emotional cues imply that all readers will necessarily read this sequence of panels in exactly the same way. What we have offered is a potential reading of interaction that combines relational and emotional cues, which shows that both go hand in hand when constructing social meaning.

Our analysis of Calvin and Susie's joint relational episode has shown that the series of panels nicely reflects the coordinated practice of relational work that is performed by the two school kids. The interactors are caught in an interaction chain of mutual social positioning and emotional evaluation. Their emotional states and appraisals cannot be seen as purely personal, internal affairs. Rather, the emotional orientations are managed by Calvin and Susie to negotiate their developing relationship and to reach their private relational goals. Along these lines, the cartoon reveals how practices of relational work are inextricably connected to semiotic acts of displaying emotions. In what follows, we would like to address some of the more specific challenges and make suggestions for future empirical work on analysing emotional displays in relational work.

One obvious reservation concerning the use of the Calvin and Hobbes cartoon to illustrate our theoretical points about the signalling of emotional orientations in relational work concerns the fictional and stylized nature of the analyzed data. At this point it is 
important to reconsider that we are dealing with a comic strip here that creates a highly comical and fictionalized account of creating an interpersonal relationship of two US school children. Our analysis can thus be questioned as it does not reflect natural interactional data. However, by way of contrast, we would like to claim that the comic strip serves our aim of illustrating the role of emotions in relational work. First, for the comic strip to work, the emotional cues must be drawn in a way that allows the reader to appreciate their evaluative impact. In other words, the displayed signals allow us to construct the fictional social world that is established between Calvin and Susie at school in a surprisingly rich way. Second, the non-conventional and rather direct use of emotional display exchanged between the two kids leads to a humorous incongruity effect with the adult world. Readers might appreciate the kids' frank use of emotional display as they are reminded of what they might often like to display but usually cannot when in a workplace (or other) situation. Indeed, Calvin and Susie's 'speech' acts do not seem to conform to the adult Anglo Western norm of emotional display rules that would be expected from the social context of collaborative work.

While the data chosen for this paper was drawn from a cartoon and from a conflict situation, we would like to suggest that the same discursive negotiations of relational meaning occur in (face-to-face) interaction of any kind. Of course, it goes without saying that our ideas will have to be further developed against more naturally occurring data. For example, similar kinds of signalling one's emotional orientation may occur in heated (political) debates on TV or private rows, which are more difficult to access and record audio-visually (see, e.g. Mondada, 2006).

When scrutinizing naturally occurring data one also has to be acutely aware of the fact that emotional display rules may suppress explicit emotional signalling in a given interaction. This, however, does not deny the role of the emotional component in interaction, which seems crucial to us and warrants further research. More precisely, one has to account for the fact that speech events are associated with unmarked, but expected conventions for emotional displays. Accordingly, we claim that the signalling of evaluations through emotional displays is present in any interactional speech event although such displays may evade our attention because they often remain unmarked. For instance, an academic lecture is associated with a certain manner of displaying one's 'neutral' and 'objective' but 'engaged' emotional orientation by adopting a specific tone of voice, making academic lexical choices, providing certain body postures and facial expressions that reflect the 'bodily hexis' (Bourdieu, 1977:90-92) of lecturing. So while not being as salient, obvious, and marked as in our Calvin and Hobbes example, these cues are still relevant for negotiating the subtle relationship between a lecturer and his/her audience.

Along these lines, we would like to argue that future empirical work will have to investigate much more deeply into the tension between unmarked, conventional and therefore expected emotional displays and highly marked ones as well as the consequences of adhering and deviating from these conventions for doing relational work. What happens in an interaction when emotional displays do not follow the expected norm? Will such deviations always trigger relational interpretations and corresponding 
communicative behaviours? What happens when emotions are under-displayed rather than exaggerated? For instance, when the receiver of a gift does not show the expected signals of joy, will this result in the giver inferring a negative evaluation of the gift and thus a rejection of him/herself as a person? And how will the giver show this to the receiver to manage their relationship communicatively? Answering such questions will provide us with a more substantial empirical basis for detailing how the emotional and the relational are connected and become visible in interactions.

Having said this, let us emphasize again that we do not claim that the full emotional world of the communicative partners is always signalled to the other. Nor do we claim that different interactors and observers will always interpret emotional cues in the same way as they are highly complex and can often only be disambiguated in context. However, we

of expected emotional displays must be considered to play an important role for the study of relational work as a discursive phenomenon.

Going beyond the level of social conventionality, we may also ask how ways of displaying emotions are associated with individual temperament and character rather than merely adhering to social and cultural norms. One can hypothesize that interactors frequently implement person-specific forms of emotional display for particular situations and that the recipients calibrate more person-based expectations against the socialconventional benchmark of appropriate emotional conduct. But where is this line between the personal and the social and how is it negotiated in actual interactions?

Finally, by drawing on Clark's differentiation between describing-as, indicating and demonstrating, we attempted to dissect the emotional element of a composite signal and to show its relevance for the creation of relational meaning in interaction. We are aware of the fact that such composite signals do not always have a straightforward and unambiguous emotional meaning but that those signals often depend on further contextual information to become disambiguated and unfold their full evaluative potential. The actual composition and patterning of composite emotional signals and their interpretation by the interactors in situ thus constitutes another pertinent area of research for a more fine-grained empirical investigation into the relationship between emotional displays and their impact on relational work.

\section{Acknowledgments}

We would like to thank the editors and the anonymous reviewers for their constructive and much valued criticism.

\section{References}

Adler, Silvia, 2011. Silence in the graphic novel. Journal of Pragmatics 43 (9), 2278-2285.

Alvarez-Pereyre, Michael, 2011. Using film as linguistic specimen: theoretical and practical issues. In: Piazza, Roberta, Bednarek, Monika, Rossi, Fabio (Eds.), Telecinematic Discourse. 
Approaches to the Language of Films and Television Series. John Benjamins, Amsterdam, pp. 47-67.

Andersen, Peter A., Guerrero, Laura K. (Eds.), 1998a. Handbook of Communication and Emotion: Research, Theory, Applications, and Contexts. Academic Press, San Diego.

Andersen, Peter A., Guerrero, Laura K., 1998b. Principles of communication and emotion in social interaction. In: Andersen, Peter A., Guerrero, Laura K. (Eds.), Handbook of Communication and Emotion: Research, Theory, Applications, and Contexts. Academic Press, San Diego, pp. 49-96.

Arundale, Robert B., 2010. Relating. In: Locher, M.A., Graham, S.L. (Eds.), Interpersonal Pragmatics. Mouton, Berlin, pp. 137-166.

Bänziger, T., Scherer, K.R., 2010. Introducing the Geneva Multimodal Emotion Portrayal (GEMEP) corpus. In: Scherer, K.R., Bänziger, T., Roesch, E.B. (Eds.), Blueprint for Affective computing: A Sourcebook. Oxford University Press, Oxford, England, pp. 271-294.

Baumeister, Roy F., Leary, Mary, 1995. The need to belong: desire for interpersonal attachments as a fundamental human motivation. Psychological Bulletin 177, 497-529.

Bourdieu, Pierre, 1977. Outline of a Theory of Practice. Cambridge University Press, Cambridge. Bråten, Stein (Ed.), 2007. On Being Moved: From Mirror Neurons to Empathy. John Benjamins, Amsterdam and Philadelphia.

Bühler, Karl H., 1934. Sprachtheorie. Fischer, Jena.

Caffi, Claudia, Janney, Richard W., 1994. Toward a pragmatics of emotive communication. Journal of Pragmatics 22 (3-4), 325-373.

Clark, Herbert H., 1996. Using Language. Cambridge University Press, Cambridge.

Couper-Kuhlen, Elizabeth, 2011. Affectivity in cross-linguistic and cross-cultural perspective. In: Stehl, Thomas (Ed.), Sprachen in mobilisierten Kultur: Aspekte der Migrationslinguistik. Universitätsverlag, Potsdam, pp. 231-257.

Culpeper, Jonathan, 2011. Impoliteness: Using Language to Cause Offence. Cambridge University Press, Cambridge.

Damasio, Antonio, 1994. Descartes' Error: Emotion, Reason, and the Human Brain. Penguin, London.

Damasio, Antonio, 1999. The Feeling of What Happens: Body and Emotion in the Making of Consciousness. Harcourt, Orlando.

Damasio, Antonio, 2003. Looking for Spinoza. Joy, Sorrow, and the Feeling Brain. Harcourt, Orlando.

Darwin, Charles, 1872. The Expression of Emotions in Man and Animals. Watts, London.

Ekman, Paul, 1973. Darwin and Facial Expression. Academic Press, New York.

Ekman, Paul, 2003. Emotions Revealed. Recognizing Faces and Feelings to Improve Communication and Emotional Life. Times Books, New York.

Ekman, Paul, Friesen, Wallace V., 1975. Unmasking the Face: A Guide to Recognizing Emotions from Facial Expressions. Prentice Hall, Englewood Cliffs, NJ.

Ekman, Paul, Friesen, Wallace V., 1978. Facial Action Coding System: A Technique for the Measurement of Facial Movement. Consulting Psychologists Press, Palo Alto, CA.

Fiehler, Reinhard, 2002. How to do emotions with words: emotionality in conversations. In: Fussell Susan, R (Ed.), The Verbal Communication of Emotions: Interdisciplinary Perspectives. Lawrence Erlbaum, Mahwah, NJ, pp. 79-106. 
Foolen, Ad, 1997. The expressive function of language. Towards a cognitive semantic approach. In: Niemeier Susanne, Driven René, (Eds.), The Language of Emotions: Conceptualization, Expression, and Theoretical Foundation. John Benjamins, Amsterdam/Philadelphia, pp. 1532.

Foolen, Ad, 2012. The relevance of emotion for language and linguistics. In: Foolen, Ad, Lüdtke, Ulrike, Racine, Tim, Zlatev, Jordan (Eds.), Moving Ourselves, Moving Others: Motion \& Emotion in Intersubjectivity, Consciousness and Language. John Benjamins, Amsterdam, pp. 349-369.

Foolen, Ad, Lüdtke, U., Racine, Tim, Zlatev, Jordan (Eds.), 2012. Moving Ourselves, Moving Others: Motion and Emotion in Intersubjectivity, Consciousness and Language. John Benjamins, Amsterdam.

Frijda, Nico H., Mesquita, Batja, 1994. The social roles and functions of emotions. In: Markus, H.R., Kitayama, S. (Eds.), Emotion and Culture. American Psychological Association, New York, pp. 51-87.

Goddard, Clifford, 2002. Explicating emotions across languages and cultures: a semantic approach. In: Fussell, S.R. (Ed.), The Verbal Communication of Emotions: Interdisciplinary Perspectives: Lawrence Erlbaum, Mahwah, NJ, pp. 19-53.

Goleman, Daniel, 1995. Emotional Intelligence: Why it Can Matter More than IQ. Bantam Books, New York.

Goodwin, Marjorie H., Goodwin, Charles, 2001. Emotion within situated activity. In: Duranti, A. (Ed.), Linguistic Anthropology: A Reader. Blackwell, Malden, MA, pp. 239-257.

Goodwin, Marjorie H., Goodwin, Charles, Yaeger-Dror, Malcah, 2002. Multimodality in girls' game disputes. Journal of Pragmatics 34, 1621-1649.

Griffiths, Paul E., 1997. What Emotions Really Are: The Problem of Psychological Categories. University of Chicago Press, Chicago.

Halliday, Michael A.K., 1975. Learning How to Mean. Edward Arnold, London. Harré, Rom (Ed.), 1986a. The Social Construction of the Emotions. Blackwell, Oxford.

Harré, Rom, 1986b. An outline of the social constructionist viewpoint. In: Harré, R. (Ed.), The Social Construction of the Emotions. Blackwell, Oxford, pp. 2-14.

Harré, Rom, Finlay-Jones, Robert, 1986. Emotion talk across times. In: Harré, R. (Ed.), The Social Construction of the Emotions. Blackwell, Oxford, pp. 220-233.

Heelas, Paul, 1996. Emotion talk across cultures. In: Harré, R., Parrot, W.G. (Eds.), The Emotions. Sage, London, pp. 171-199.

Hochschild, Arlie R., 1979. Emotion work, feeling rules, and social structure. American Journal of Sociology 85, 551-575.

Hochschild, Arlie R., 1983. The Managed Heart: Commercialization of Human Feeling. University of California Press, Berkeley.

Izard, Caroll E., 1971. The Face of Emotion. Appleton-Century-Crofts, New York.

Jakobson, Roman, 1960. Closing statements: linguistics and poetics. In: Seboek, Th.A. (Ed.), Style In Language. MIT Press, Cambridge, MA, pp. 350-377.

Jay, Timothy, Janschewitz, Kristin, 2007. Filling the emotion gap in linguistic theory: commentary of Potts' expressive dimension. Theoretical Linguisitics 33 (2), 215-221.

Jing-Schmidt, Zhou, 2007. Negativity bias in language: a cognitive-affective model of emotive intensifiers. Cognitive Linguistics 18 (3), 417-443. 
Jones, Rodney, 2013. Multimodal discourse analysis. In: Chapelle, Carol E. (Ed.), The Encyclopedia of Applied Linguistics. Wiley-Blackwell, Oxford, UK.

Kimmich, Mathias, 2008. Disorienting visualisations: adapting Paul Auster's City of Glass. SPELL: Swiss Papers in English Language and Literature 21, 87-104.

Kress, Gunther, van Leeuwen, Theo, 1996. Reading Images: The Grammar of Visual Design. Routledge, London.

Langlotz, Andreas, 2010. Social cognition. In: Locher, M.A., Graham, S.L. (Eds.), Interpersonal Pragmatics. Mouton, Berlin, pp. 167-202.

Languages of Emotion Cluster of Excellence at the Freie Universität Berlin. Available from: http://www.languages-of-emotion.de/ (accessed 27.01.13).

LeVine, Philip, Scollon, Ron (Eds.), 2004. Discourse and Technology: Multimodal Discourse Analysis. Georgetown University Press, Washington, DC.

Locher, Miriam A., 2004. Power and Politeness in Action: Disagreements in Oral Communication. Mouton de Gruyter, Berlin.

Locher, Miriam A., 2008. Relational work, politeness and identity construction. In: Antos, G., Ventola, E., Weber, T. (Eds.), Handbooks of Applied Linguistics. Vol. 2: Interpersonal Communication. Mouton de Gruyter, Berlin, pp. 509-540.

Locher, Miriam A., 2012. Politeness research from past to future, with a special focus on the discursive approach. In: Lucía, Fernandez Amaya, Lopez, Maria de la O. Hernandez, Reyes, Gomez Moron, Manuel, Padilla Cruz, Manuel, Mejias Borrero, Mariana, Relinque Barranca (Eds.), New Perspectives on (Im)Politeness and Interpersonal Communication. Cambridge Scholars Publishing, Cambridge, pp. 1-22.

Locher, Miriam A., 2013. Politeness. In: Chapelle, Carol E. (Ed.), The Encyclopedia of Applied Linguistics. Wiley-Blackwell, Oxford, UK.

Locher, Miriam A., Graham, Sage Lambert, 2010. Introduction to interpersonal pragmatics. In: Locher, M.A., Graham, S.L. (Eds.), Interpersonal Pragmatics. Mouton, Berlin, pp. 1-13.

Locher, Miriam A., Langlotz, Andreas, 2008. Relational work: at the intersection of cognition, interaction and emotion. Bulletin Suisse de Linguistique Appliquée (VALS-ASLA) 88, 165-191. Locher, Miriam A., Watts, Richard J., 2005. Politeness theory and relational work. Journal of Politeness Research 1, 9-33.

Locher, Miriam A., Watts, Richard J., 2008. Relational work and impoliteness: negotiating norms of linguistic behaviour. In: Bousfield, D., Locher, M.A. (Eds.), Impoliteness in Language: Studies on its Interplay with Power in Theory and Practice. Mouton de Gruyter, Berlin, pp. 77-99.

LPRG - Linguistic Politeness Research Group (Eds.), 2011. Discursive Approaches to Politeness. Walter de Gruyter, Berlin.

Malloch, Stephen, Trevarthen, Colwyn (Eds.), 2009. Communicative Musicality. Exploring the Basis of Human Companionship. Oxford University Press, Oxford.

Matoesian, Greg, 2005. Struck by speech revisited: embodied stance in jurisdictional discourse. Journal of Sociolinguistics 9 (2), 167-193.

McKeon, Richard, 1941. The Basic Works of Aristotle. Random House, New York.

Michel, Caroline, Caldara, Roberto, Rossion, Bruno, 2006. Same-race faces are perceived more holistically than other-race faces. Visual Cognition 14 (1), 55-73.

Mondada, Lorenza, 2006. Video recording as the reflexive preservation and configuration of phenomenal features for analysis. In: Knoblauch, H., Raab, J., Soeffner, H.-G., Schnettler, B. 
(Eds.), Video Analysis. Methodology and Methods. Qualitative Audiovisual Data Analysis in Sociology. Peter Lang, Bern, pp. 51-67.

Morsbach, Helmut, Tyler, William J., 1986. A Japanese emotion: Amae. In: Harré, R. (Ed.), 1986.

The Social Construction of the Emotions. Blackwell, Oxford, pp. 289-307.

Niemeier, Susanne, Dirven, René (Eds.), 1997. The Language of Emotions: Conceptualization,

Expressions, and the Theoretical Foundation. John Benjamins, Amsterdam.

Nishdia, Tracy K., Lillard, Angeline S., 2007. The informative value of emotional expressions.

Developmental Science 10 (2), 205-212.

Norris, Sigrid, 2004. Analyzing Multimodal Interaction: A Methodological Framework. Routledge, New York.

Ochs, Elinor, 1986. From feelings to grammar: a Samoan case study. In: Schieffelin, B., Ochs, E.

(Eds.), Language Socialization Across Cultures. Cambridge University Press, Cambridge, pp.

251-272.

Ochs, Elinor, Schieffelin, Bambi, 1984. Language acquisition and socialization: three developmental stories and their implications. In: Shweder, R., Le Vine, R.A. (Eds.), Culture Theory: Essays on Mind, Self, and Emotion. Cambridge University Press, New York, pp. 276320.

Ochs, Elinor, Schieffelin, Bambi, 1989. Language has a heart. Text 9 (1 (special issue: The Pragmatics of Affect)), 7-25.

Oster, U., 2010. Using corpus methodology for semantic and pragmatic analyses: what can corpora tell us about the linguistic expression of emotions? Cognitive Linguistics 21 (4), 727763.

Peirce, Charles S., 1977. Semiotics and Significs. Ed. by Charles Hardwick. Indiana University Press, Bloomington, IN.

Pepin, Nicolas (Ed.), 2008. Studies on Emotions in Social Interactions. Special issue of the Bulletin Suisse de Linguistique Appliquée (VALS-ASLA) 88. Centre de linguistique appliquée, Université de Neuchâtel, Neuchâtel. Available from:

http://doc.rero.ch/Im.php?url=1000,20,10,bulletin_vals_asla_2008_088 pdf.

Planalp, Sally, 1998. Communicating emotion in everyday life: cues, channels, and processes. In: Andersen, P.A., Guerrero, L.K. (Eds.), Handbook of Communication and Emotion: Research, Theory, Applications, and Contexts. Academic Press, San Diego, pp. 29-48.

Planalp, Sally, 1999. Communicating Emotion: Social, Moral, and Cultural Processes. Cambridge University Press, Cambridge.

Planalp, Sally, Knie, Karen, 2002. Integrating verbal and nonverbal emotion(al) messages. In: Fussell, Susan R. (Ed.), The Verbal Communication of Emotions: Interdisciplinary Perspectives. Lawrence Erlbaum, Mahwah, New Jersey, pp. 55-77.

Plutchik, Robert, 2003. Emotions and Life: Perspectives from Psychology, Biology, and Evolution. American Psychological Association, Washington.

Reber, Elisabeth, 2012. Affectivity in Interaction. Sound Objects in English. John Benjamins, Amsterdam.

Rozin, Paul, Royzman, Edward B., 2001. Negativity bias, negativity dominance, and contagion. Personality and Social Psychology Review 5 (4), 296-320.

Ruhi, Sükriye, 2009. Evoking face in self and other-presentation in Turkish. In: Bargiela-Chiappini, Francesca, Haugh, Michael (Eds.), Face, Communication and Social Interaction. Equinox, London, pp. 155-174. 
Saraceni, Mario, 2003. The Language of Comics. Inter Text. Routledge, London.

Schwarz-Friesel, Monika, 2007. Sprache und Emotion. Francke, Tübingen.

Spencer-Oatey, Helen, 2007. Theories of identity and the analysis of face. Journal of Pragmatics 39 (4), 639-656.

Spencer-Oatey, Helen, 2011. Conceptualising 'the relational' in pragmatics: insights from metapragmatic emotion and (im)politeness comments. Journal of Pragmatics 43 (14), 35653578.

Spencer-Oatey, Helen, 2013. Relating at work: facets, dialectics and face. Journal of Pragmatics, http://dx.doi.org/10.1016/j.pragma.2013.02.010.

Tannen, Deborah, 1993. What's in a frame? Surface evidence for underlying expectations. In: Tannen, D. (Ed.), Framing in Discourse. Oxford University Press, Oxford, pp. 14-56.

Tomasello, Michael, Carpenter, Malinda, Call, Josep, Behne, Tanya, Moll, Henrike, 2005. Understanding and sharing intentions: the origins of cultural cognition. Behavioral and Brain Sciences 28, 675-729.

Tomkins, Silvan S., 1962. Affect, Imagery, and Consciousness, Vol. 1: The Positive Affects. Springer, New York.

Tomkins, Silvan S., 1963. Affect, Imagery, and Consciousness, Vol. 2: The Negative Affects. Springer, New York.

Tronick, Edward Z., 2005. Why is connection with others so critical? The formation of dyadic states of consciousness: coherence governed selection and the co-creation of meaning out of messy meaning making. In: Nadel, Jacqueline, Muir, Darwin (Eds.), Emotional Development. Oxford University Press, Oxford, pp. 293-315.

Turner, Jonathan H., 2000. On the Origins of Human Emotions. A Sociological Enquiry into the Evolution of Human Affect. Stanford University Press, Stanford.

Turner, Jonathan H., 2007. Human Emotions: A Sociological Theory. Routledge, Oxford.

Turner, Jonathan H., Stets, Jan E., 2005. The Sociology of Emotions. Cambridge University Press, Cambridge.

Watterson, Bill, 1990. The Authoritative Calvin and Hobbes. Warner Books, London. Wierzbicka, Anna, 1994. Emotion, language, and cultural scripts. In: Kitayama, S., Marcus, H.R. (Eds.), Emotion and Culture. American Psychological Association, Washington, DC, pp. 133196.

Wilce, James E., 2009. Language and Emotion. Cambridge University Press, Cambridge. 